\title{
Knockdown adiponectin receptor 1 expression in synovial tissue alleviate the progression of collagen-induced arthritis
}

\section{Yani Wang}

The First Affiliated Hospital of Nanjing Medical University

Rui Liu

The First Affiliated Hospital of Nanjing Medical University

Pengfei Zhao

The First Affiliated Hospital of Nanjing Medical University

Qian Zhang

The First Affiliated Hospital of Nanjing Medical University

\section{Yingheng Huang}

The First Affiliated Hospital of Nanjing Medical University

\section{Lei Wang}

The First Affiliated Hospital of Nanjing Medical University

\section{Chengyin Lv}

The First Affiliated Hospital of Nanjing Medical University

\section{Nan Che}

The First Affiliated Hospital of Nanjing Medical University

\section{Wenfeng Tan}

The First Affiliated Hospital of Nanjing Medical University

Miaojia Zhang ( $\sim$ mjzhang@njmu.edu.cn )

The First Affiliated Hospital of Nanjing Medical University

\section{Research article}

Keywords: Rheumatoid arthritis, Adiponectin, Adiponectin receptor 1, synovial fibroblasts, bone erosion

Posted Date: December 30th, 2019

DOI: https://doi.org/10.21203/rs.2.19703/v1

License: (1) (1) This work is licensed under a Creative Commons Attribution 4.0 International License.

Read Full License 


\section{Knockdown adiponectin receptor 1 expression in synovial tissue alleviate the}

progression of collagen-induced arthritis

Yani Wang ${ }^{1 \#}$, Rui Liu ${ }^{1 \#}$, Pengfei Zhao ${ }^{1}$, Qian Zhang ${ }^{1}$, Yingheng Huang ${ }^{1}$, Lei Wang ${ }^{1}$,

Chengyin Lv ${ }^{1}$, Nan Che ${ }^{1}$, Wenfeng Tan ${ }^{1}$, Miaojia Zhang ${ }^{1 *}$

${ }^{1}$ Department of Rheumatology, The First Affiliated Hospital of Nanjing Medical University, 300 Guangzhou Road, Nanjing 210029, China.

\#These authors contributed equally to this work.

${ }^{*}$ Corresponding authors:

Prof. Miaojia Zhang E-mail address: mjzhang@njmu.edu.cn.

Department of Rheumatology, The First Affiliated Hospital of Nanjing Medical University, 300 Guangzhou Road, Nanjing 210029, China. 


\begin{abstract}
Background: Previous studies have shown that adiponectin (APN) is involved in the pathogenesis of rheumatoid arthritis (RA). The proinflammatory effect of APN is mainly mediated adiponectin receptor 1 (AdipoR1). The high expression of AdipoR1 have been suggested in RA synovial tissue. This study was aimed to investigate the effects of AdipoR1 in inflammation and bone erosion in collagen-induced arthritis (CIA) mice, and to further explore the underlying mechanisms.
\end{abstract}

Methods: The expression of APN and AdipoR1 in synovial tissue of RA and osteoarthritis (OA) patient was tested by qPCR and western blot. RA synovial fibroblasts (RASFs) were stimulated with APN, IL-6 or TNF- $\alpha$ respectively. The expression of AdipoR1 on RASFs were tested by flow cytometry. To prove the pathogenic role, AdipoR1 was silenced in a human rheumatoid arthritis synovial fibroblast cell line (MH7A) and local joint of CIA mice by specific short hairpin RNAs (shRNAs) using a lentiviral delivery system. The levels of proliferation, apoptosis and inflammatory factors on MH7A were assessed in vitro. Local AdipoR1 knockdown on CIA mice were further estimated by arthritis clinical scores, inflammatory cytokine expression, micro-CT, H\&E staining and receptor activator of nuclear factor к В ligand (RANKL) / osteoprotegerin (OPG) in vivo.

Results: We found that the levels of APN and AdipoR1 expression were significantly higher in RASFs and the expression of AdipoR1 was upregulated by APN in RASFs. Silencing AdipoR1 could effectively reduce lipopolysaccharides (LPS) induced proliferation of MH7A cells, promote their apoptosis, and reduce the release of inflammatory factors. In CIA mice, local silencing AdipoR1 in arthritis markedly reduced joint inflammation and alleviated bone 
erosion and osteoporosis in vivo. Furthermore, local silencing AdipoR1 inhibited receptor activator of nuclear factor $\mathrm{\kappa} B$ ligand (RANKL) expression and decreased RANKL / osteoprotegerin (OPG) ratio in knees and ankles of CIA mice.

Conclusions: This study suggests that AdipoR1 plays a key role in the development of RA and silencing AdipoR1 might be a new target for the clinical treatment of RA.

Keywords: Rheumatoid arthritis, Adiponectin, Adiponectin receptor 1, synovial fibroblasts, bone erosion 


\section{Background}

Rheumatoid arthritis (RA) is a chronic inflammatory disorder and irreversible destructive arthropathy. The inflammation is mainly driven by the overproduction of pivotal proinflammatory cytokines, including tumour necrosis factor (TNF)- $\alpha$, interleukin (IL)-1 $\beta$, and IL-6[1]. These cytokines mediate long-term cartilage degradation and bone erosion, resulting in joint pain and dysfunction[2]. Notably, evidence suggests that rheumatoid arthritis synovial fibroblasts (RASFs) are key integrators of inflammatory signals in the inflamed rheumatoid synovium[3]. One of the most significant features of RASFs is destroying cartilage, and RASFs are considered as the primary cells responsible for marginal cartilage destruction by secretion of matrix-degrading enzymes and inflammatory cytokines[4].

Accumulating evidences demonstrated that APN could promote the secretion of IL-1 IL-6, vascular cell adhesion molecule (VCAM)-1 and exacerbate erosive joint destruction in RA[5-7]. Recently, our group found that APN induced joint destruction through promoting osteopontin (OPN) expression in RASFs[8], and the overexpression of APN could promote the differentiation of Thelper cell 17 (Th17 cell) and enhance the expression of RANKL thus exacerbating symptoms of collagen-induced arthritis (CIA) model[9]. It indicated that APN might be involved in the pathogenesis of RA. There are two main types of APN receptors, AdipoR1 and AdipoR2, that mediated its biological effects[10]. We found a high concentrations of adiponectin (APN) and the expression levels of adiponectin receptor 1 (AdipoR1) in RA patients [11], indicating that AdipoR1 might be the effector receptor. Considering the dynamic effects of APN in vivo, directly knockdown its effector receptors might be an ideal strategy to intervene the local inflammation in the joint. 
In present study, we intended to reveal relationships among APN, AdipoR1 and the inflammatory factors in synovial tissue of RA patients. Lentivirus-mediated technology was used to detect whether AdipoR1 knockdown could decrease the inflammatory responses in MH7A in vitro and delay the disease progression in collagen-induced arthritis (CIA) mice in vivo. This study aims to get insights into the pathogenic mechanism of AdipoR1 and provide the potential therapeutic targets for the treatments of RA. 


\section{Materials and methods}

\section{Patients}

RA and Osteoarthritis (OA) patients and the healthy controls (HC) were recruited randomly from the First Affiliated Hospital of Nanjing Medical University as our previously described[8]. In short, the synovial tissues were obtained from 3 patients with RA and 3 patients with OA that fulfilled the American College of Rheumatology Criteria for RA or OA. This study was approved by the Ethics Committee at the First Affiliated Hospital of Nanjing Medical University, and all donors signed informed consent forms.

\section{RNA extraction and Quantitative real-time PCR}

RNA samples were isolated from collected cells using Trizol reagent (Invitrogen). RNA was converted to cDNA using Prime Script ${ }^{\mathrm{TM}}$ RT regent Kit (Takara) according to the manufacturer's instructions. PCR amplification and analysis were achieved using an ABI 7900 system (ABI), and the cycling parameters were performed an initial denaturation step at $95^{\circ} \mathrm{C}$ for 10 minutes, followed by 40 cycles at $95^{\circ} \mathrm{C}$ for 15 seconds and extension $60^{\circ} \mathrm{C}$ for 1 minute. PCR primers used for real-time PCR were presented in Supplement Table S1. Relative gene expression was determined by $2^{-\Delta \Delta \mathrm{Ct}}$ method.

\section{Protein extraction and western blotting analysis}

Cells were solubilized with RIPA lysis buffer (Thermo Fisher Scientific, Inc.) according to the manufacturer's instructions and protein concentration was detected using a BCA kit (Thermo Fisher Scientific, Inc.). Approximately $50 \mu \mathrm{g}$ of protein from each sample was separated by SDS-PAGE gels and electrophoretically transferred to PVDF membrane (Bio-Rad, USA). Then, the PVDF membrane were blocked with 5\% skim fat dry milk in 
Tris-buffered saline (TBS) with $0.1 \%$ Tween-20 for $2 \mathrm{~h}$ at room temperature to block the non-specific sites on blots. The membranes were incubated with primary antibodies including APN (1:1000; Abcam), AdipoR1 (1:1000; Abcam), RANKL (1:1000; Abcam), IL-6 (1:500; Abcam) and GAPDH (1:2000, Cell Signaling) overnight at $4^{\circ} \mathrm{C}$ and then incubated with an HRP-labeled secondary antibody (1:2000; Cell Signaling) for $1 \mathrm{~h}$ at room temperature. GAPDH was used as a loading control. Proteins were detected with the SuperSignal West Pico Chemiluminescent Kit (Thermo Scientific). Densitometry values were analyzed and quantified with ImageLab.

\section{Flow cytometry}

Single-cell suspensions from RASFs were prepared. All samples were treated according to the manufacturer's recommendations. RASFs were stained with purified AdipoR1(Abcam) at a dilution of 1:300 for $30 \mathrm{~min}$. The secondary antibody used was Alexa Fluor 647 Donkey Anti-Rabbit IgG (1:2000; Abcam).

\section{Cell culture}

Primary RA synovial fibroblasts (RASFs) were isolated from the synovial tissue of patients with RA. The cells used for the experiments were at the 3 to 6 passage. RASFs were starved for 12 hours before adding APN in serum-free Dulbecco's modified Eagle's medium (DMEM; Gibco). Then cells were cultured in DMEM supplemented with $10 \%$ fetal bovine serum (Gibco) in the presence of APN and pro-inflammatory cytokines: APN (10 $\mu \mathrm{g} / \mathrm{ml}$; ProperTech), TNF- $\alpha$ (50 ng/ml; ProperTech) and IL-6 (50 ng/ml; ProperTech), then incubated for 48 hours in 12 -well plates at a density of $5 \times 10^{5}$ cells $/$ well at $37^{\circ} \mathrm{C}$. The human $\mathrm{RA}$ synovial cell line MH7A used in gene transfection experiments was procured from Dr. Seiichi 
Tanuma (Tokyo University of Science). Cells were maintained in RPMI 1640 medium (Hyclone; GE Healthcare, Logan, UT, USA) containing 10\% fetal bovine serum (Gibco) and 1\% penicillin/streptomycin (1:100, Sigma-Aldrich; Merck KGaA, Darmstadt, Germany), at $37^{\circ} \mathrm{C}$ in $95 \%$ humidified incubator under $5 \% \mathrm{CO}_{2}$. The LPS induced concentration was $100 \mathrm{ng} / \mathrm{mL}$.

\section{Knockdown AdipoR1 in MH7A cell line}

Lentiviral particles carrying the AdipoR1 short hairpin RNA (Lenti-shAdipoR1) were purchased from Genechem (Shanghai, China). The lentiviral vectors were produced by cotransfection of 293T kidney cells. MH7A were cultured in 6-well plates at an inoculation density of $5 \times 10^{4}$ cells/well and infected with a lentivirus containing shAdipoR1 or control shRNA (shNC). After incubation for $72 \mathrm{~h}$, the infection efficiency was observed using fluorescence microscopy and analysed by qPCR and Western blotting.

\section{Cell counting kit 8 (CCK-8) assay}

MH7A cells were plated in 96-well plates with the density of 5,000 cells/well for $72 \mathrm{~h}$ transfected with shAdipoR1 or corresponding controls (shNC) for $24 \mathrm{~h}$. Cells were treated with lipopolysaccharide (LPS) at room temperature to construct a model of rheumatoid arthritis. The control group were treated with normal RPMI 1640 medium. CCK-8 solution (Invitrogen; Thermo Fisher Scientific, Inc.) was added to the wells after $12 \mathrm{~h}, 24 \mathrm{~h}, 48 \mathrm{~h}$, separately and samples were incubated at $37^{\circ} \mathrm{C}$ for $2 \mathrm{~h}$. The absorbance of each well was measured at $450 \mathrm{~nm}$ using a microplate reader.

\section{Flow cytometric analysis of apoptosis}

Cells were seeded in the 6-well plate at a concentration of $1 \times 10^{6} / \mathrm{ml}$, treated with LPS 
and were harvested after transfection with AdipoR1 for $48 \mathrm{~h}$. Cells were washed twice with cold PBS and resuspended in $100 \mu$ binding buffer. Annexin V and propidium iodide (PI) double-staining was carried out using an Annexin V-FITC/PI apoptosis detection kit (BD Biosciences, San Jose, CA, USA) according to manufacturer's instructions. Apoptosis analysis was performed using the BD LSRII Flow Cytometer System.

\section{Induction of collagen-induced arthritis (CIA) mice and treatment}

All animal experimental protocols were approved by the animal care and use committee of the First Affiliated Hospital of Nanjing Medical University and we confirmed that all methods were performed in accordance with the relevant guidelines and regulations. Male DBA/1 J mice (aged 8-12 weeks) were purchased from Shanghai Laboratory Animal Center, Chinese Academy of Science. Experiments divided in four groups:NC, CIA mice, shAdipoR1 treated CIA mice and shNC treated CIA mice. CIA mice were induced as following described. In Brief, $100 \mu \mathrm{g}$ of bovine collagen type II (CII; Chondrex, Redmond, WA, USA) that was dissolved in $0.05 \mathrm{M}$ acetic acid was emulsified with an equal volume of Freund's complete adjuvant (Difco, Detroit, MI, USA) and administered intradermally at the base of tail into DBA/1 J mice. On day 21 after the initial immunization, a booster immunization was administered intradermally near the primary injection site. Simultaneously, the lentivirus-mediated shAdipoR1 $\left(10^{7} \mathrm{TU}\right)$ was intraarticularly injected into the knee joints of shAdipoR1 treated CIA mice and the lentivirus-mediated $\operatorname{shNC}\left(10^{7} \mathrm{TU}\right)$ was injected into the knee joints of shNC treated CIA mice, and the other knees were treated with an equivalent volume of PBS as controls. Mice were examined by two blinded observers daily after the 2nd CII immunization for the signs of arthritis. The arthritis score was evaluated by grading each 
paw from 0 (no inflammation) to 4 based on erythema, swelling, and flexion of the joint and the clinical score was constructed by adding the scores from the 4 individual paws of each CIA mouse.

\section{Haematoxylin \& eosin (H\&E) staining}

The ankles or paws were removed from euthanized mice for histopathological examination after routine fixation, decalcification, and paraffin embedding of the tissue. The tissues were then sectioned into $3-\mu \mathrm{m}$ slices, deparaffinized in xylene, rehydrated using a series of ethanol concentrations and stained with haematoxylin and eosin(H\&E). The slides were evaluated for synovial hyperplasia, inflammatory infiltration pannus formation, and cartilage/subchondral bone destruction by two blinded observers. Each joint was scored from 0 to 4. Briefly, grade 0 , no signs of inflammation; grade 1, mild inflammation with hyperplasia of the synovial lining without cartilage destruction; grades 2 to 4 , increasing degrees of inflammatory cell infiltrate and cartilage/bone destruction.

\section{Micro-computed tomography (Micro-CT) analysis}

On day 45 post the 1st immunization, mice were sacrificed and knee joints and ankles were removed and fixed in 4\% paraformaldehyde. Quantitative analysis of knee joints and ankles was performed using a Micro-CT Scan SkyScan1176S scanner at a resolution of $9 \mu \mathrm{m}$. Computed tomographic images of the knee joints and ankles of the mice in all four groups $(n=3)$ were acquired. After scanning, cross-sectional slices were reconstructed. Three-dimensional models of the knee joints and paws were performed to verify the bone destruction, using SkyScan CT Analyzer version 1.8. Cortical bone mineral density, bone mass, bone surface/volume ratio, trabecularbone mineral density, percent bone volume and 
structural model index were performed using scanner software (Skyscan CTAn).

\section{Statistical analysis}

For statistical evaluation, results are expressed as Mean \pm SD. Student's $t$ test was performed to assess the statistic difference between two groups. The statistic difference among three or more groups were evaluated by one-way analysis of variance (ANOVA) followed by Tukey's Multiple Comparison Test. All results were considered statistically significant if the $\mathrm{p}$ value is $<0.05$. 


\section{Results}

\section{The expression of AdipoR1 was upregulated by APN in RASFs}

Firstly, we detected the expression of APN and AdipoR1 in synovial tissue of RA patients. The result showed that the levels of APN and AdipoR1 mRNA expression were significantly higher in RA group, compared with OA group (Figure 1A, $P<0.05$, n=3). These differences were also observed in protein levels (Figure 1B, $P<0.05, \mathrm{n}=3$ ). Furthermore, to find the reason of high expressed of AdipoR1 in RASF, primary RA synovial fibroblasts (RASFs) was stimulated with APN $(10 \mu \mathrm{g} / \mathrm{ml})$ or IL-6 $(50 \mathrm{ng} / \mathrm{ml})$ or TNF- $\alpha(50 \mathrm{ng} / \mathrm{ml})$ respectively. Flow cytometry showed that the frequency of AdipoR1 was significantly higher in APN stimulated RASFs group $(1.73 \pm 0.20 v s 1.00 \pm 0.07, P<0.01)$. There was no significant difference among IL-6, TNF- $\alpha$ and NC groups (Figure 1C). These results indicated that the expression of AdipoR1 was significantly higher in RASFs, and it was upregulated by the treatment of APN. AdipoR1 might function as a downstream signaling molecule of APN and play an important role in the micro-environmental changes of RA joints.

\section{AdipoR1 knockdown prevented MH7A responses}

To further determine the pathogenic role of AdipoR1 in RA, the expression of adipoR1 in MH7A was knocked down by shRNA/GFP lentivirus infection. Green fluorescent protein (GFP) expression in MH7A infected by the lentivirus vectors for $72 \mathrm{~h}$ showed over $80 \%$ infection efficiency (Figure S1A). The gene and protein expression levels also proved that AdipoR1 was successful knockdown (n=6, $P<0.01$, Figure S1B and C). The result showed that the proliferation of MH7A was significantly decreased and the apoptosis of MH7A was increased after AdipoR1 knockdown ( $P<0.05$, Figure 2A, B and C). In addition, AdipoR1 
knockdown substantially suppressed the expression of IL-1 $\beta$, IL-6 and TNF- $\alpha$ in MH7A induced by LPS (Figure 2D). Therefore, these results suggested that APN-AdipoR1 pathway might be involved in RASFs proliferation, apoptosis, and pro-inflammatory release in RA.

\section{AdipoR1 knockdown alleviated the progression of collagen-induced arthritis (CIA) mice}

To further assess the effects of AdipoR1 on the process of inflammation in vivo, the lentivirus-mediated shAdipoR1 was injected into the knee joints of CIA mice on 21st day post the first immunization. AdipoR1 knockdown delayed onsets of arthritis and exhibited lower clinical scores CIA mice treated with shNC (Figure 3A). Both the gene and protein expression of AdipoR1 in synovial tissue were significantly decreased in shAdipoR1 treated group (Figure 3B, C). Haematoxylin \& eosin (H\&E) staining also revealed that AdipoR1 knockdown improved pronounced synovial hyperplasia, decreased the synovial hyperplasia and inflammatory infiltration pannus formation in CIA mice (Figure 3D). Histopathological analysis on H\&E staining indicated shAdipoR1 group showed significantly lower scores than control groups (Figure 3E). Inflammatory cytokine such as IL-1 $\beta$, IL-6 and IL-17 expressions were remarkably decreased in AdipoR1 knockdown group (Figure 3F). Our results demonstrated that AdipoR1 knockdown could alleviate inflammation and improve the pathological process in vivo.

\section{Local AdipoR1 knockdown prevented bone destruction by suppressing RANKL expression in CIA mice}

Osteoclastogenesis induced by inflammatory cytokines contributes to focal and systemic bone and cartilage destruction including secondary osteoporosis in RA. To further verify the effects of AdipoR1 on bone erosion, the local joints features of knee, ankle and trabecular 
bone were evaluated by Micro-CT. Representative three-dimensional renditions indicated that AdipoR1 local knockdown treatment attenuated the damage in knee, ankle, and trabecular bone in CIA mice (Figure 4A). Compared to shNC group, the cortical bone mineral density and bone volume were ascending, while the ratios of bone surface / volume were descending both in knee and ankle (Figure 4B, C). Besides, local inhibition of AdipoR1 pathway raised the trabecular bone density and percent bone volume and degraded the structure model index (Figure 4D). The activation of RANKL have been proved tightly associated with osteoclast development [12]. Expectedly, shAdipoR1 group had both lower RANKL expression level and RANKL / osteoprotegerin (OPG) ratio compared to shNC group (Figure 4E, F). It suggested that AdipoR1 knockdown treatment decreased joint erosion through suppressed RANKL/OPG axis via inhibiting the expression of RANKL. Taken together, our results confirmed that local AdipoR1 knockdown decrease synovial inflammation, joint erosion and osteoporosis progression in CIA mice. 


\section{Discussion}

RA is characterized as the presence of an inflammatory synovitis accompanied by destruction of joint cartilage and bone. Although the underlying mechanism stay unknown, several studies find that multiple proinflammatory cytokines play critical roles in the pathophysiology of RA, including IL-1 $\beta$, IL-6, TNF- $\alpha$ and so on[13]. However, the initiating factors of these inflammatory responses are still unclear.

APN was reported to be rather detrimental in RA and involved in disease progression, by inducing the secretion of pro-inflammatory molecules, chemokines and matrix-degrading enzymes $[6,14,15]$. There are two main types of APN receptors, AdipoR1 and AdipoR2, that mediated the biological effects[10]. In present study, AdipoR1 was found high-expressed in synovial tissue of RA patients. Moreover, the expression of AdipoR1 was upregulated after APN stimulation in primary RASFs. It suggested that AdipoR1 mediated the downstream changes, and it might be a therapeutic target in the treatment of RA.

RASFs mediate most relevant pathways of joint destruction. Molecular insights into these cells constitute an important target for novel therapeutic approaches that inhibit the destruction of cartilage and bone in RA[16]. When activated by LPS, RASFs could elaborate a broad repertoire of inflammatory mediators, justifying their classification as immune sentinel cells in RA[17]. In MH7A, AdipoR1 knockdown significantly suppressed the abnormal proliferation stimulated by LPS. Besides, evidence suggests that the expansion of fibroblast populations in the joint results primarily from inhibition of pro-apoptotic pathways, rather than large-scale proliferation[18]. In present study, AdipoR1 knockdown promoted the apoptosis and inhibited the inflammatory factors secretion of MH7A, suggesting a therapeutic 
effect in vitro.

In previously studies, we reported APN exacerbate disease progression, by enhancing Th17 response, prompting RANKL expression and osteopontin production[8, 9]. Since RASFs play a central role in the progression of RA, lentivirus mediated shAdipoR1 was injected into the joints of CIA mice to achieve local AdipoR1 knockdown. The outcomes indicated that it significantly improved the pathological process, inflammatory reactions and bone erosion. In RA, bone erosions result from osteoclastic bone resorption at the sites of synovitis. RANKL plays a critical role in the bone homeostasis, which could be elevated by several cytokines[19]. RANKL/OPG axis determines the degree of proliferation and activity of the osteoclasts. Interestingly, local AdipoR1 knockdown could prevent CIA mouse from bone erosion by repressing the expression of RANKL without affecting the expression of OPG. It reveals a distinct mechanism of cartilage destruction and bone erosion in CIA mouse, and it provides us a novel way to block bone erosion in arthritis.

Current pharmacologic therapies have made a significant progress toward achieving disease remission without joint deformity. Despite this, a significant proportion of RA patients do not effectively respond to the current therapies and thus new drugs are urgently required[18]. Biological disease-modifying anti-rheumatic drugs (DMARDs) had revolutionized RA treatment. Current DMARDs targets a variety of receptors, such as Infliximab targets TNF- $\alpha[20]$, Anakinra targets IL-1[4], Tocilizumab targets IL-6[21], Ixekizumab targets RANKL[22] and so on. APN is widely distributed and plays systemically protective effects on human, including metabolic homeostasis, preventing cardiovascular diseases and so on[23]. Paradoxically, adiponectin has been proved to have both positive and 
negative influences in the inflammation mechanism of RA. In mice, systemic APN knock out aggravated the condition of arthritis [24]. Furthermore, APN significantly exacerbated the symptoms of RA in local joints[9]. Thereby, local AdipoR1 suppression might be an ideal target for the discovery of biological DMARDs in future.

\section{Conclusions}

In summary, we demonstrated that AdipoR1 knockdown treatment could decrease the inflammatory responses, improve the phenotype of RASFs and suppress the progression of RA through inhibiting RANKL expression. Our study reveals a new sight into the pathophysiological process of RA and provides a potential therapeutic target in the treatment of RA. 


\section{List of Abbreviations}

APN, adiponectin; RA, rheumatoid arthritis; AdipoR1, adiponectin receptor 1; MH7A, a human rheumatoid arthritis synovial fibroblast cell line; CIA, collagen-induced arthritis; shRNAs, short hairpin RNAs; LPS, lipopolysaccharides; RANKL, receptor activator of nuclear factor к В ligand; OPG, osteoprotegerin; TNF- $\alpha$, tumour necrosis factor; IL, interleukin; RASFs, rheumatoid arthritis synovial fibroblasts; OPN, osteopontin; AdipoR2, adiponectin receptor 2; Th17 cell, T helper cell 17; OA, osteoarthritis; HC, healthy controls; Lenti-shAdipoR1, lentiviral particles carrying the AdipoR1 short hairpin RNA; shNC, control shRNA; CCK-8, cell counting kit 8; PI, propidium iodide; CII, bovine collagen type II; H\&E, haematoxylin \& eosin; Micro-CT, micro-computed tomography; ANOVA, one-way analysis of variance; GFP, green fluorescent protein; RANK, receptor activator of nuclear factor к B; KO, knock out; DMARDs, disease-modifying anti-rheumatic drugs; O.D., optical density. 


\section{Declarations}

\section{Ethics approval and consent to participate}

This study was approved by the Ethics Committee at the First Affiliated Hospital of Nanjing Medical University, and all donors signed written informed consent forms. The animal experiments were conducted in compliance with the guidelines for the care and use of laboratory animals and approved by Institutional Animal Care and Use Committee of Nanjing Medical University.

\section{Consent for publication}

Not applicable.

\section{Availability of data and materials}

Not applicable.

\section{Competing interests}

The authors declare no conflict of interest.

\section{Funding}

This study was supported by National Natural Science Foundation of China (NSFC) (81671615, 81771758 and 81501393), the Special Project of Clinical Medicine from Jiangsu Province (BL2013034). 


\section{Authors' contributions}

Miaojia Zhang and Wenfeng Tan conceived and designed the experiments. Yani Wang, Rui Liu, Pengfei Zhao, Qian Zhang, Yingheng Huang, Lei Wang performed experiments. Chengyin Lv and Nan Che collected samples. Ya'ni Wang and Rui Liu wrote the manuscript. All authors read and approved the final manuscript.

\section{Acknowledgements}

Not applicable.

\section{Authors' information}

Department of Rheumatology, The First Affiliated Hospital of Nanjing Medical University, 300 Guangzhou Road, Nanjing 210029, China.

\section{Endnotes}

Not applicable. 


\section{References}

1. Brennan FM, McInnes IB: Evidence that cytokines play a role in rheumatoid arthritis. The Journal of Clinical Investigation 2008, 118(11):3537-3545.

2. Norma A, Jessica GM, Ledesma-Colunga MG, Perales-Canales SI, Andrés QS, Fernando LB, Isabel M, Bibiana MC, Jakob $\mathrm{T}$, Nadine B: Prolactin promotes cartilage survival and attenuates inflammation in inflammatory arthritis. Journal of Clinical Investigation 2013, 123(9):3902-3913.

3. Huber LC, Distler O, Tarner I, Gay RE, Gay S, Pap T: Synovial fibroblasts: key players in rheumatoid arthritis. Rheumatology 2006, 45(6):669-675.

4. Cavalli G, Dinarello CA: Treating rheumatological diseases and co-morbidities with interleukin-1 blocking therapies. Rheumatology 2015, 54(12):2134-2144.

5. Liu $\mathrm{D}$, Luo $\mathrm{S}$, Li $\mathrm{Z}$ : Multifaceted roles of adiponectin in rheumatoid arthritis. International Immunopharmacology 2015, 28(2):1084-1090.

6. Ebina K, Fukuhara A, Ando W, Hirao M, Koga T, Oshima K, Matsuda M, Maeda K, Nakamura T, Ochi T: Serum adiponectin concentrations correlate with severity of rheumatoid arthritis evaluated by extent of joint destruction. Clinical Rheumatology 2009, 28(4):445-451.

7. Kim KS, Choi HM, Da HO, Kim C, Jin SJ, Yoo MC, Yang HI: Effect of taurine chloramine on the production of matrix metalloproteinases (MMPs) in adiponectin- or

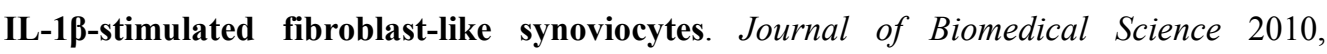
17(S1):1-7.

8. Qian J, Xu L, Sun X, Wang Y, Xuan W, Zhang Q, Zhao P, Wu Q, Liu R, Che N: Adiponectin aggravates bone erosion by promoting osteopontin production in synovial tissue of rheumatoid arthritis. Arthritis Research \& Therapy 2018, 20(1):26.

9. Sun X, Feng X, Tan W, Lin N, Hua M, Wei Y, Wang F, Li N, Zhang M: Adiponectin exacerbates collagen-induced arthritis via enhancing Th17 response and prompting RANKL expression. Scientific Reports 2015, 5:11296.

10. Toshimasa Y, Junji K, Yusuke I, Atsushi T, Takehiko Y, Shunbun K, Takuya S, Makoto M, Kazuo H, Masaki T: Cloning of adiponectin receptors that mediate antidiabetic metabolic effects. Nature 2007, 423(6941):762.

11. Tan W, Wang F, Zhang M, Guo D, Zhang Q, He S: High Adiponectin and Adiponectin Receptor 1 Expression in Synovial Fluids and Synovial Tissues of Patients with Rheumatoid Arthritis. Semin Arthritis Rheum 2009, 38(6):420-427.

12. Lacey DL, Boyle WJ, Simonet WS, Kostenuik PJ, Dougall WC, Sullivan JK, Martin JS, Dansey R: Bench to bedside: elucidation of the OPG-RANK-RANKL pathway and the development of denosumab. Nature Reviews Drug Discovery 2012, 11:401.

13. Chen Z, Bozec A, Ramming A, Schett G: Anti-inflammatory and immune-regulatory cytokines in rheumatoid arthritis. Nat Rev Rheumatol 2019, 15(1):9-17.

14. Giles JT, Heijde DM, Van Der, Bathon JM: Association of circulating adiponectin levels with progression of radiographic joint destruction in rheumatoid arthritis. Annals of the Rheumatic Diseases 2011, 70(9):1562. 
15. Klein-Wieringa IR, Linden MPM, Van Der, Rachel K, Kwekkeboom JC, Els VB, Huizinga TWJ, Annette HVM, Margreet K, Toes REM, Andreea IF: Baseline serum adipokine levels predict radiographic progression in early rheumatoid arthritis. Arthritis \& Rheumatism 2014, 63(9):2567-2574.

16. Huber LC, Distler O, ., Tarner I, ., Gay RE, Gay S, ., Pap T, . Synovial fibroblasts: key players in rheumatoid arthritis. Rheumatology 2006, 45(6):669.

17. Turner JD, Andrew F: The role of the synovial fibroblast in rheumatoid arthritis pathogenesis. Current Opinion in Rheumatology 2015, 27(2):175.

18. Guo Q, Wang $\mathrm{Y}, \mathrm{Xu} \mathrm{D}$ : Rheumatoid arthritis: pathological mechanisms and modern pharmacologic therapies. Bone Research 2018, 6(1).

19. Weitzmann MN: The Role of Inflammatory Cytokines, the RANKL/OPG Axis, and the Immunoskeletal Interface in Physiological Bone Turnover and Osteoporosis. Scientifica,2013,(2013-2-3) 2013, 2013(3):125705.

20. Kim EY, Moudgil KD: Immunomodulation of autoimmune arthritis by pro-inflammatory cytokines. Cytokine 2017, 98:87-96.

21. Raimondo MG, Biggioggero M, Crotti C, Becciolini A, Favalli EG: Profile of sarilumab and its potential in the treatment of rheumatoid arthritis. Drug Des Devel Ther 2017, 11:1593-1603.

22. Fassio A, Rossini M, Viapiana O, Idolazzi L, Benini C, Vantaggiato E, Gatti D: New strategies for the prevention and treatment of systemic and local bone loss; from pathophysiology to clinical application. Curr Pharm Des 2017, 23(41).

23. Stern J, Rutkowski J, Scherer P: Adiponectin, Leptin, and Fatty Acids in the Maintenance of Metabolic Homeostasis through Adipose Tissue Crosstalk. Cell Metabolism 2016, 23(5):770-784.

24. Fantuzzi G: Adiponectin in inflammatory and immune-mediated diseases. Cytokine 2013, 64(1):1-10. 


\section{Figure legends}

Figure 1 The expression of APN and AdipoR1 was upregulated in RASFs

(A) The gene expression levels of APN and AdipoR1 in RA group were significantly higher than those in OA group $(n=3)$. (B) The protein expression levels of APN and AdipoR1 in RA group were significantly higher than those in OA group. (C) The primary RASF were stimulated with TNF- $\alpha$, IL-6, and APN for $24 \mathrm{~h}$. The proportion of AdipoR1 on RASF was significantly higher after APN stimulation group than other groups. The experiments were performed 3 times with similar results. Values are presented as the means \pm S.D. ${ }^{*} P<0.05$, ${ }^{* *} P<0.01$, compared to NC group.

Figure 2 AdipoR1 knockdown reduced the inflammatory responses in MH7A induced by

\section{LPS}

(A) The proliferation rate of MH7A was promoted by LPS stimulation, and inhibited by AdipoR1 knockdown. **, Compared with shNC group, the O.D. of MH7A was significant higher, $P<0.01, \mathrm{n}=6$. \#, Compared with LPS + shNC group, the O.D. of MH7A was significant higher, $P<0.05, \mathrm{n}=6$. $(\mathbf{B}, \mathbf{C})$ AdipoR1 knockdown promoted the apoptosis of MH7A induced by LPS. Compared with LPS+shNC group, the apoptosis of LPS+shAdipoR1 group was significantly higher. ${ }^{* * *} P<0.005$, compared to shNC group. (D) AdipoR1 knockdown significantly inhibited the gene expression of IL-1 $\beta$, IL-6 and TNF- $\alpha$ from MH7A (n=6). ${ }^{*} P<0.05,{ }^{* *} P<0.01$, compared to shNC group. The experiments were performed 3 times with similar results. Values are presented as the means \pm S.D.

Figure 3 Local AdipoR1 knockdown suppressed the progression of joint inflammation in

\section{CIA mice}


(A) AdipoR1 knockdown delayed onsets of arthritis and exhibited lower clinical scores than CIA mice treated with shNC ( $\mathrm{n}=12)$. (B) The gene expression of AdipoR1 was significantly decreased in synovial tissue after lentivirus-mediated shAdipoR1 injection. ${ }^{*} P<0.05$, compared to shNC group. (C) The protein expression of AdipoR1 was significantly decreased in synovial tissue after lentivirus-mediated shAdipoR1 injection. (D) H\&E staining of knee and ankle joint showed decreased synovial hyperplasia and inflammatory infiltration pannus formation after local AdipoR1 knockdown. (E) The histopathological scores of knee and ankle were quantitative analyzed. (F) Local AdipoR1 knockdown significantly decreased the gene expression levels of IL-1 $\beta$, IL- 6 and IL-17. Values are presented as the means \pm S.D. ${ }^{*} P<0.05,{ }^{* *} P<0.01$, compared with NC group.

Figure 4 Local AdipoR1 knockdown alleviated bone erosion and osteoporosis in CIA mice

(A) On day 45, the three-dimensional renditions of the ankles, knee and trabecular bone were scanned by microcomputed tomography (Micro-CT). The cortical bone mineral density, bone volume and the ratio of bone surface / volume of knee (B) and ankle (C) were calculated. (D) The trabecular bone density, percent bone volume and structure model index of trabecular bone were calculated. (E) The gene expression levels of RANKL, OPG and RANKL/OPG were detected by qPCR. (F) The protein expression level of RANKL was observed by western blot. The experiments were performed 3 times with similar results. Values are presented as the means \pm S.D. ${ }^{*} P<0.05,{ }^{* *} P<0.01$, compared with $\mathrm{NC}$ group. 
Figures

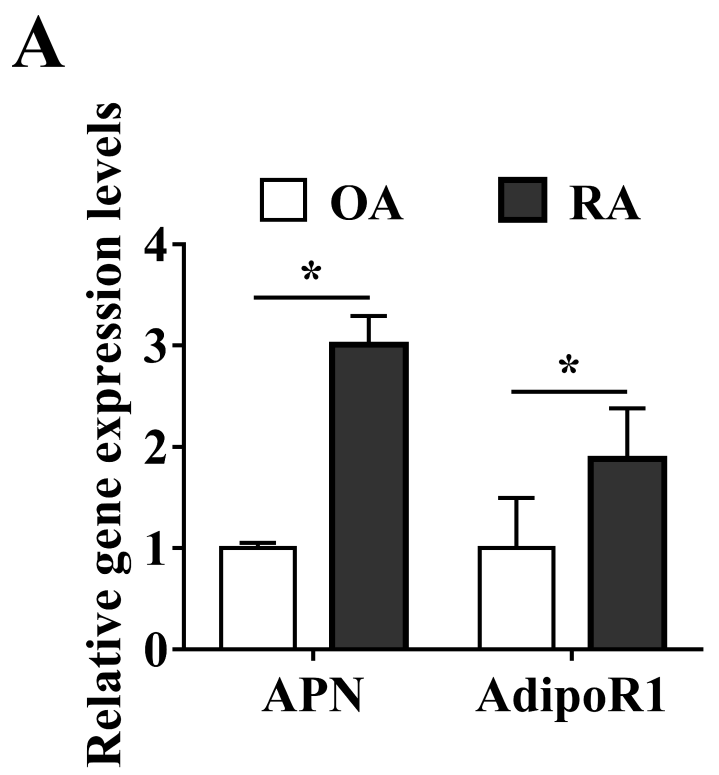

B

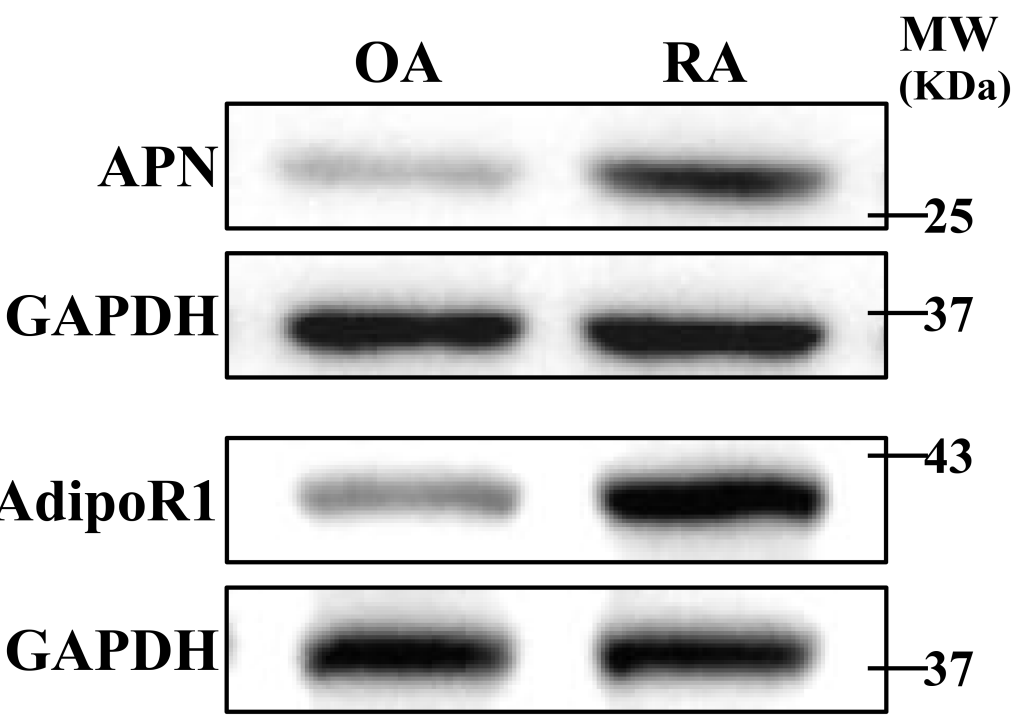

C
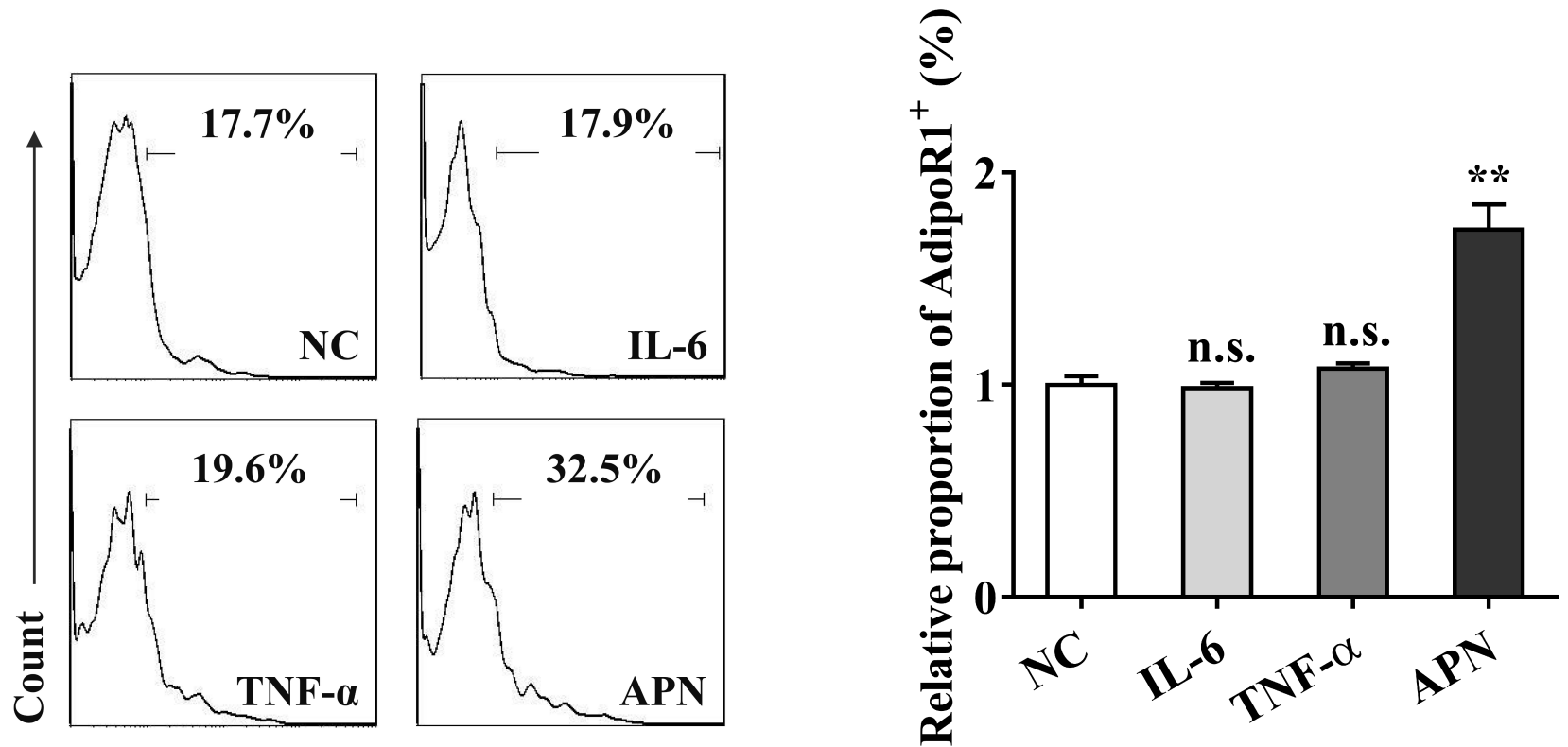

\section{AdipoR1}

Figure 1

The expression of APN and AdipoR1 was upregulated in RASFs (A) The gene expression levels of APN and AdipoR1 in RA group were significantly higher than those in OA group $(n=3)$. (B) The protein expression levels of APN and AdipoR1 in RA group were significantly higher than those in OA group. (C) The primary RASF were stimulated with TNF-a, IL-6, and APN for $24 \mathrm{~h}$. The proportion of AdipoR1 on RASF was significantly higher after APN stimulation group than other groups. The experiments were performed 3 times with similar results. Values are presented as the means \pm S.D. ${ }^{*} P<0.05,{ }^{\star}{ }^{*}<<0.01$, compared to NC group. 

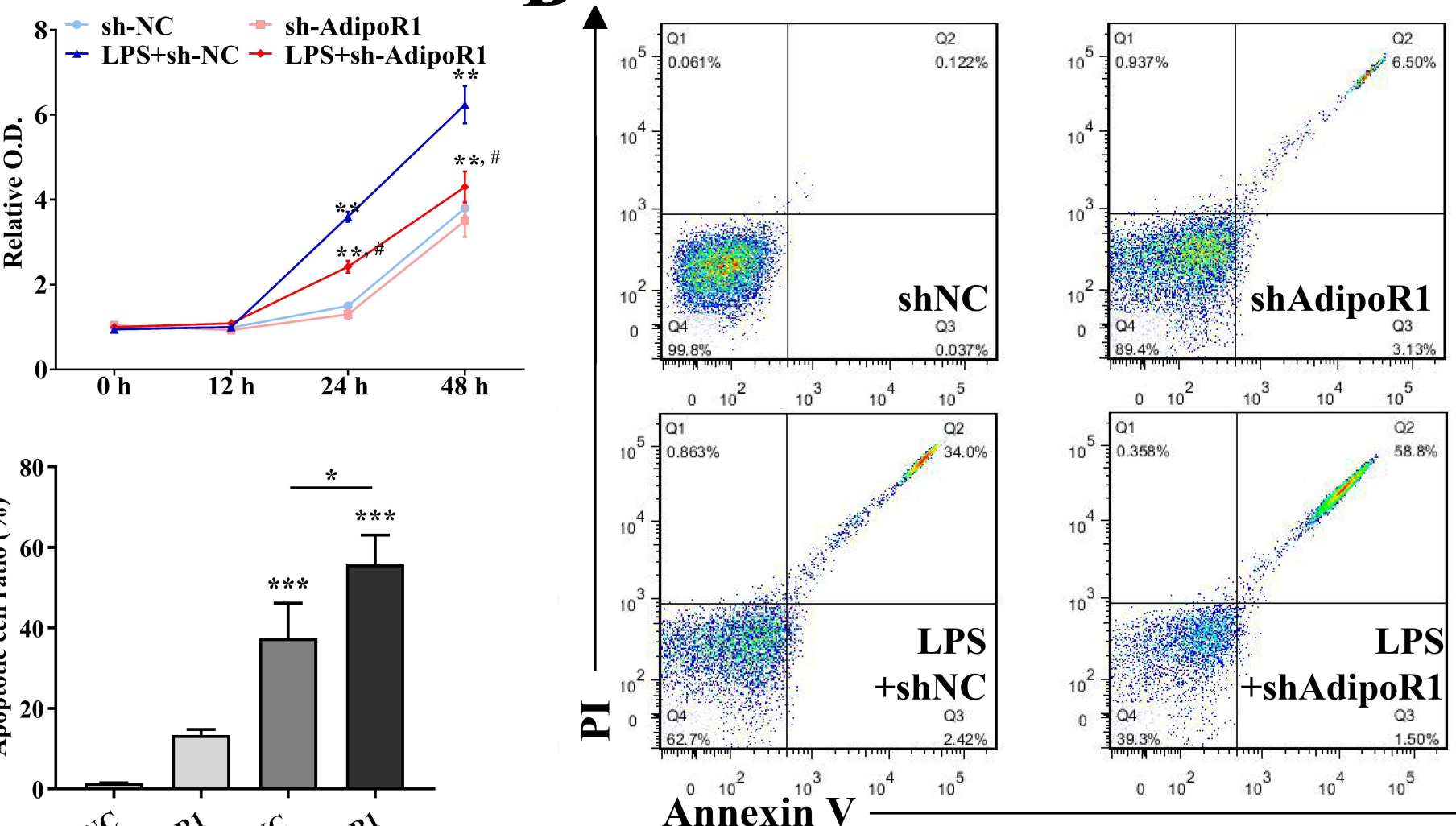

C
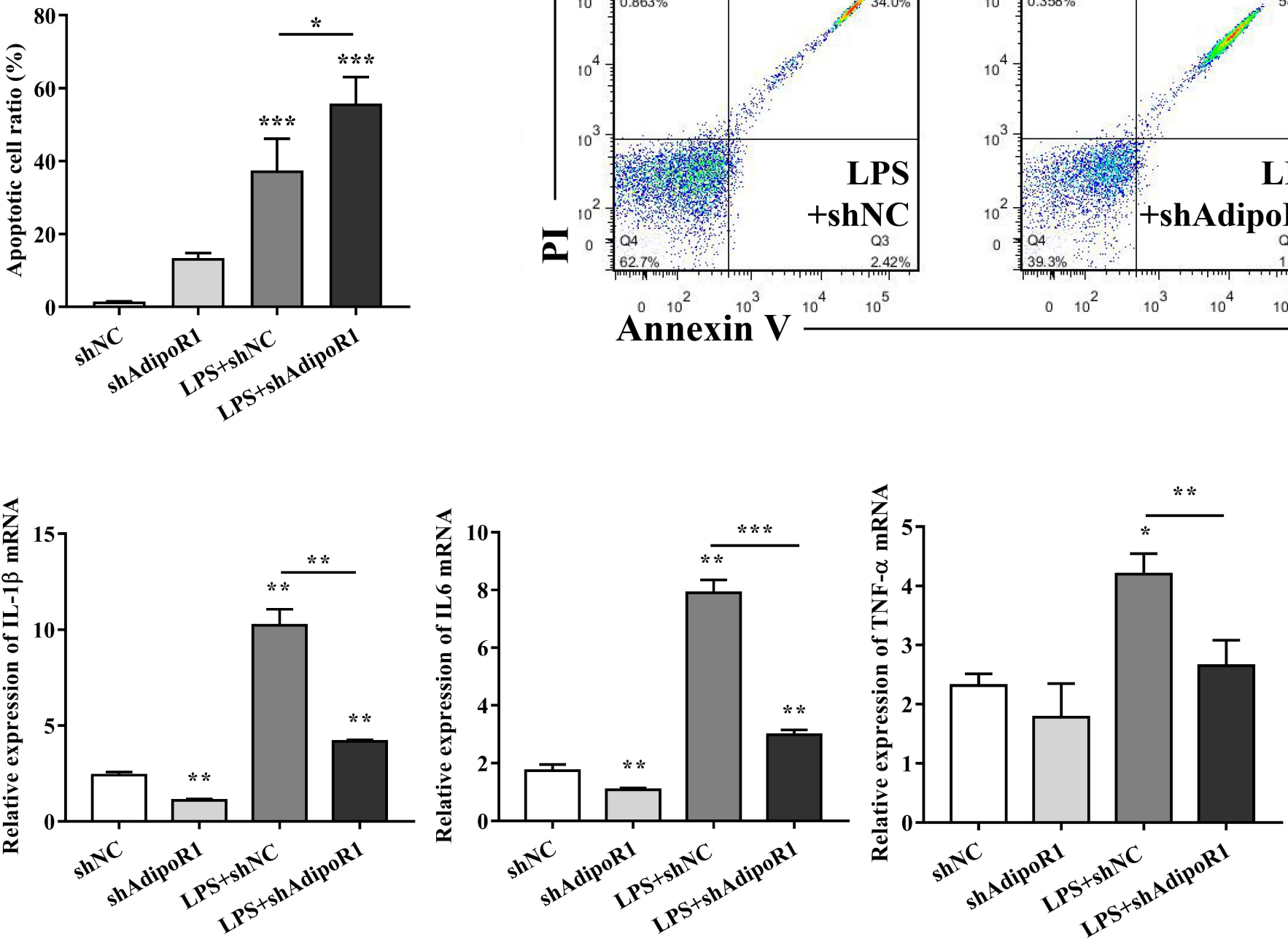

Figure 2

AdipoR1 knockdown reduced the inflammatory responses in MH7A induced by LPS (A) The proliferation rate of MH7A was promoted by LPS stimulation, and inhibited by AdipoR1 knockdown. **, Compared with shNC group, the O.D. of MH7A was significant higher, $P \otimes 0.01, n=6$. \#, Compared with LPS+shNC group, the O.D. of MH7A was significant higher, $P \unrhd 0.05, n=6$. (B, C) AdipoR1 knockdown promoted the apoptosis of MH7A induced by LPS. Compared with LPS+shNC group, the apoptosis of LPS+shAdipoR1 group was significantly higher. ${ }^{*} * \mathrm{P}<0.005$, compared to shNC group. (D) AdipoR1 knockdown significantly inhibited 
the gene expression of IL- $1 \beta$, IL- 6 and TNF-a from MH7A $(n=6) .{ }^{*} P<0.05,{ }^{*} P<0.01$, compared to shNC group. The experiments were performed 3 times with similar results. Values are presented as the means \pm S.D.

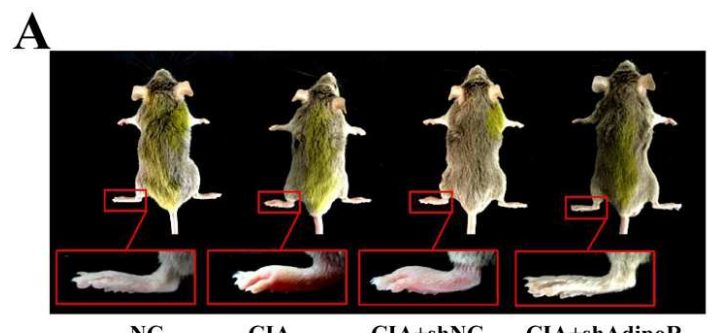

B

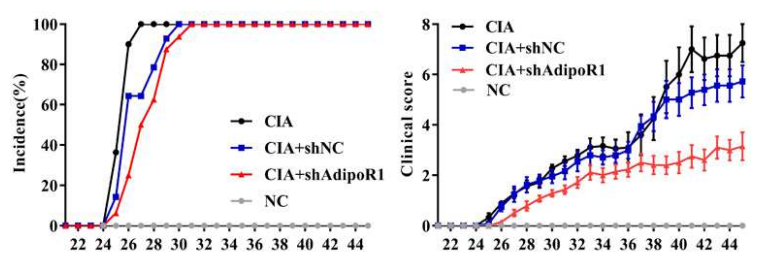

C Days post $1^{\text {st }}$ immunization Days post $1^{\text {st }}$ immunization
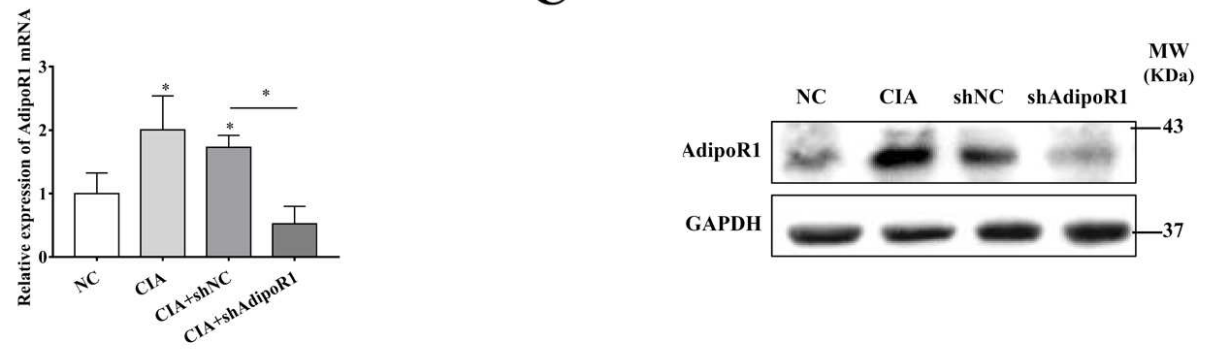

D

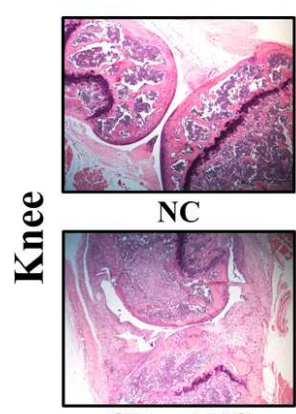

CIA +shNC

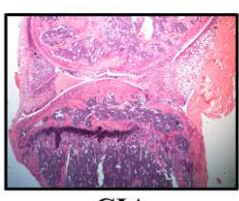

CIA

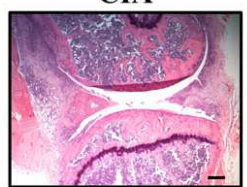

CIA+shAdipoR1

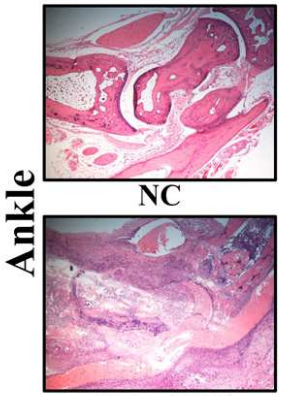

CIA+shNC

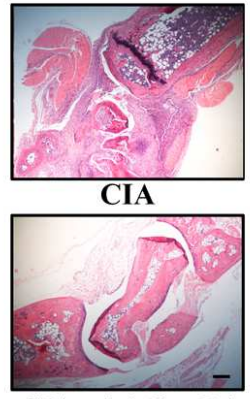

CIA+shAdipoR1

$\mathbf{E}$

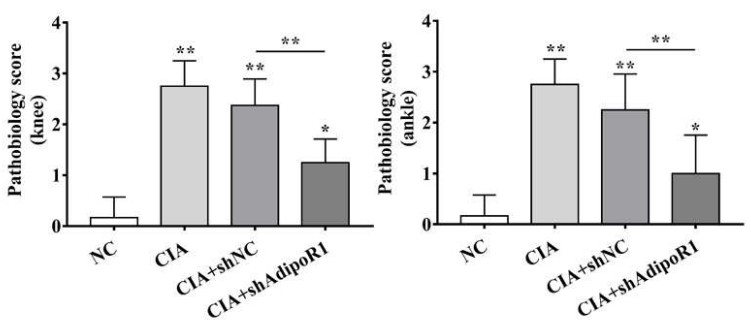

$\mathbf{F}$
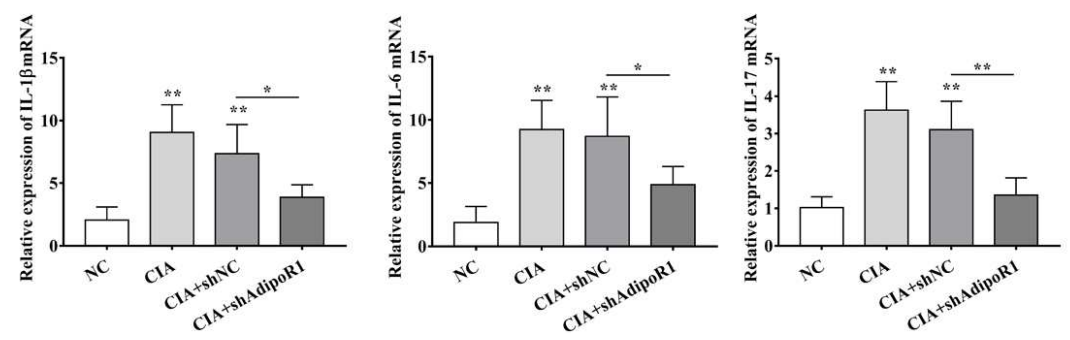

Figure 3

Local AdipoR1 knockdown suppressed the progression of joint inflammation in CIA mice (A) AdipoR1 knockdown delayed onsets of arthritis and exhibited lower clinical scores than CIA mice treated with 
shNC $(n=12)$. (B) The gene expression of AdipoR1 was significantly decreased in synovial tissue after lentivirus-mediated shAdipoR1 injection. ${ }^{\star} \mathrm{P} \otimes 0.05$, compared to shNC group. (C) The protein expression of AdipoR1 was significantly decreased in synovial tissue after lentivirus-mediated shAdipoR1 injection. (D) H\&E staining of knee and ankle joint showed decreased synovial hyperplasia and inflammatory infiltration pannus formation after local AdipoR1 knockdown. (E) The histopathological scores of knee and ankle were quantitative analyzed. (F) Local AdipoR1 knockdown significantly decreased the gene expression levels of IL-1 $\beta$, IL- 6 and IL-17. Values are presented as the means \pm S.D. ${ }^{*} P<0.05,{ }^{*} P<<0.01$, compared with NC group. 
A

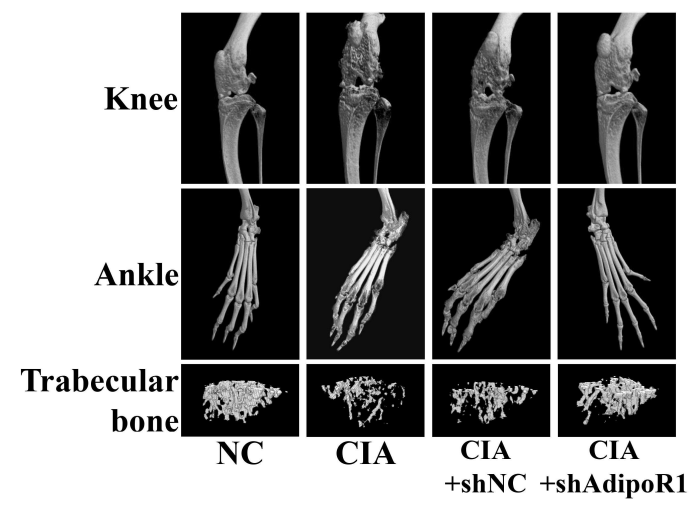

B
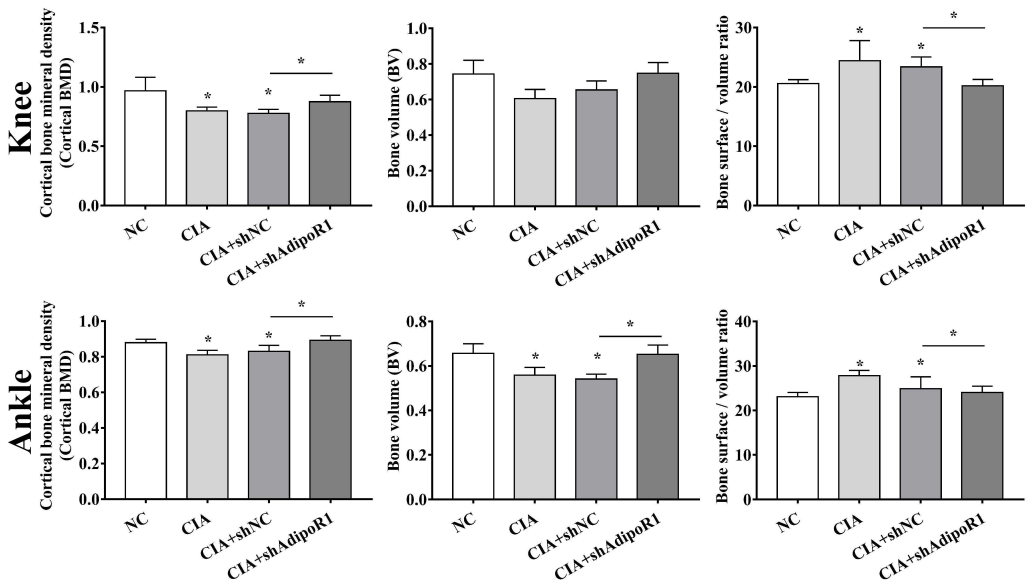

D
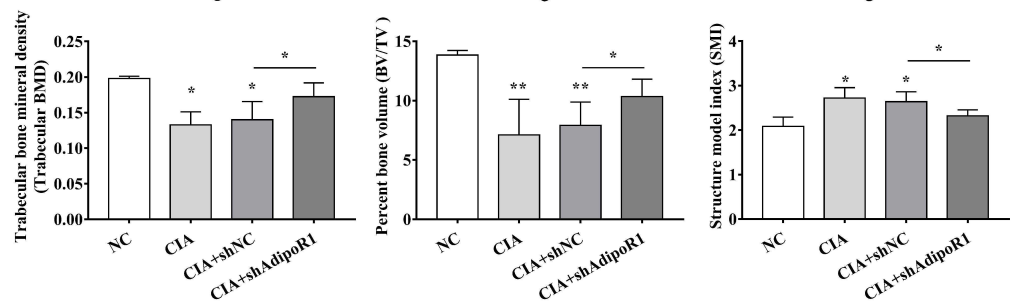

$\mathbf{E}$
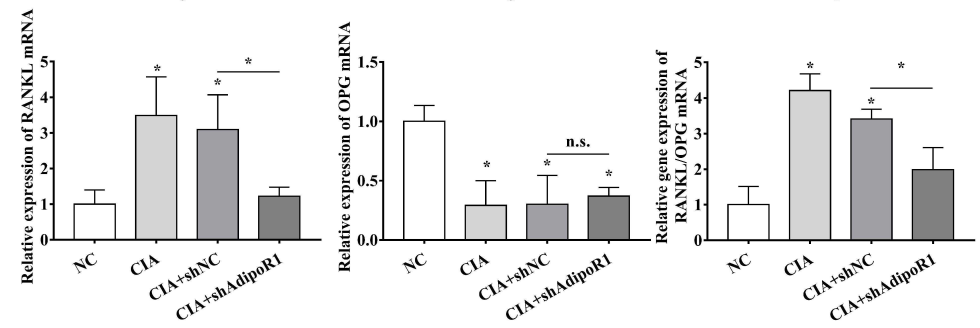

$\mathbf{F}$

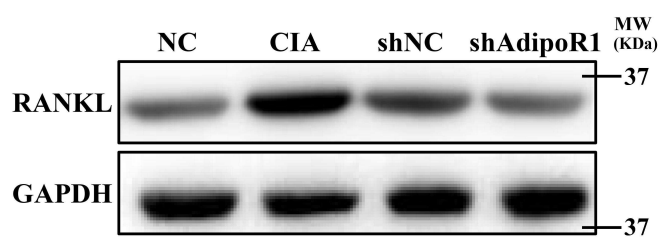

\section{Figure 4}

Local AdipoR1 knockdown alleviated bone erosion and osteoporosis in CIA mice (A) On day 45, the threedimensional renditions of the ankles, knee and trabecular bone were scanned by microcomputed tomography (Micro-CT). The cortical bone mineral density, bone volume and the ratio of bone surface / volume of knee (B) and ankle (C) were calculated. (D) The trabecular bone density, percent bone volume and structure model index of trabecular bone were calculated. (E) The gene expression levels of RANKL, 
OPG and RANKL/OPG were detected by qPCR. (F) The protein expression level of RANKL was observed by western blot. The experiments were performed 3 times with similar results. Values are presented as the means \pm S.D. ${ }^{*} \mathrm{P}<0.05,{ }^{*} \mathrm{P}<0.01$, compared with $\mathrm{NC}$ group.

\section{Supplementary Files}

This is a list of supplementary files associated with this preprint. Click to download.

- Supplement.pdf 